\title{
Perioperative safety and feasibility outcomes of stage IIIA-N2 non-small cell lung cancer following neoadjuvant immunotherapy or neoadjuvant chemotherapy: a retrospective study
}

\author{
Zhangfeng Huang ${ }^{1}$, Zhe Wu ${ }^{1}$, Yi Qin ${ }^{1}$, Yandong Zhao ${ }^{1}$, Yunpeng Xuan ${ }^{1}$, Tong Qiu ${ }^{1}$, Ao Liu ${ }^{1}$, \\ Yanting Dong ${ }^{1}$, Wenhao Su ${ }^{1}$, Wenxing Du ${ }^{1}$, Tianxiang Yun ${ }^{1}$, Lingjie Wang ${ }^{1}$, Dahai Liu ${ }^{1}$, Lili Sun ${ }^{2}$, \\ Wenjie Jiao ${ }^{1}$ \\ ${ }^{1}$ Department of Thoracic Surgery, Affiliated Hospital of Qingdao University, Qingdao, China; ${ }^{2}$ Department of Ultrasound, First Affiliated Hospital \\ of Jinzhou Medical University, Jinzhou, China \\ Contributions: (I) Conception and design: Z Huang, Z Wu, Y Qin; (II) Administrative support: Y Zhao, Y Xuan, T Qiu; (III) Provision of study \\ materials or patients: A Liu, Y Dong; (IV) Collection and assembly of data: W Su, W Du, T Yun; (V) Data analysis and interpretation: L Wang, D \\ Liu, L Sun; (VI) Manuscript writing: All authors; (VII) Final approval of manuscript: All authors. \\ Correspondence to: Wenjie Jiao. Department of Thoracic Surgery, Affiliated Hospital of Qingdao University, Qingdao, China. \\ Email: jiaowj@qduhospital.cn.
}

Background: We sought to determine the perioperative safety and feasibility outcomes of stage IIIA (N2) non-small cell lung cancer (NSCLC) following neoadjuvant immunotherapy or neoadjuvant chemotherapy.

Methods: The clinical details of patients who attended the Affiliated Hospital of Qingdao University between January 2019 and December 2020 were retrospectively evaluated. Eligible patients had pathologically proven stage IIIA (N2) NSCLC and were randomly prescribed neoadjuvant therapy. Those in the neoadjuvant immunotherapy group received two cycles of nivolumab $(3 \mathrm{mg} / \mathrm{kg})$ and those in the control group received neoadjuvant chemotherapy $\left(1,000 \mathrm{mg} / \mathrm{m}^{2}\right.$ gemcitabine and $80 \mathrm{mg} / \mathrm{m}^{2}$ cisplatin). All patients were scheduled to undergo surgery. The primary endpoint was the risk of major complications within 30 days of surgery and the secondary endpoints were interval to surgery and 30-day mortality.

Results: A total of 107 eligible patients were evaluated of whom 25 were allocated to the neoadjuvant immunotherapy group and 82 to the neoadjuvant chemotherapy group. The median interval to surgery was similar in the two groups at 29.2 days [95\% confidence interval (CI), 27.1 to 31.4 days] in the immunotherapy group and 28.7 days (95\% CI, 27.6 to 29.8 days) in the chemotherapy group ( $\mathrm{P}=0.656)$. While treatmentrelated adverse events were reported in most patients, all 25 patients completed two cycles of neoadjuvant immunotherapy and 80 of 82 patients completed two cycles of neoadjuvant chemotherapy, although one patient in the latter group died within 30 days of surgery. There was no statistically significant difference between the groups in the probability of grade 3 or higher postoperative complications within 30 days after surgery $(\mathrm{P}=0.757)$.

Conclusions: Most patients achieved the primary and secondary endpoints of the study. However, the major pathological response (MPR) showed statistically significant differences between the neoadjuvant immunotherapy and neoadjuvant chemotherapy groups.

Keywords: Non-small cell lung cancer (NSCLC); neoadjuvant therapy; IIIA-N2 disease; perioperative; safety and feasibility

Submitted Feb 05, 2021. Accepted for publication Apr 17, 2021.

doi: $10.21037 / \mathrm{atm}-21-1141$

View this article at: http://dx.doi.org/10.21037/atm-21-1141 


\section{Introduction}

Lung cancer carries the highest morbidity and mortality of all malignancies globally (1). The proportion of lung cancer with non-small cell lung cancer (NSCLC) histology exceeds $80 \%$, and about $20 \%$ of NSCLC patients have reached stage IIIA (N2) disease at the time of initial treatment (2). The prognosis of NSCLC patients is closely related to the stage of the disease at the time of initial diagnosis, and early-stage patients are usually defined as stage I, II, and some stage IIIA (N2) patients who can undergo surgical excision. Surgery is the most effective treatment strategy and the cornerstone of all standard treatments for NSCLC, and is the most effective treatment in patients diagnosed with stage IIIA (N2) (3). The 5-year overall survival (OS) in early-stage patients can reach 36-92\% after radical surgical resection treatment, while in late stage patients the 5 -year OS is less than $10 \%(4,5)$. However, the OS among patients who undergo surgical resection alone is not optimal as the existence of preoperative micro-metastases increases the risk of recurrence and metastasis of patients after surgery (3). On the other hand, patients with locally advanced tumors (T2-T4) or localized lymph node involvement (N1, single-level N2) have benefited from multimodal therapy, including platinum-based adjuvant chemotherapy after surgery.

Therefore, a large number of researchers have begun to explore neoadjuvant therapy strategies for NSCLC in recent years, and this has proven to be an effective method to prolong the OS of patients and reduce the probability of recurrence (6). In 2014, the NSCLC Metaanalysis Collaborative Group published a meta-analysis in the "Lancet" journal showing that NSCLC neoadjuvant chemotherapy had a significant survival benefit compared with surgery alone, and the 5 -year OS benefit increased by $5 \%$ (from $40 \%$ to $45 \%$ ) $(7,8)$. In addition, multiple clinical trials including the NATCH and CSLC0501 have obtained negative results, which proves that it is feasible and safe to choose adjuvant chemotherapy before or after surgery $(9,10)$. Until now, the recommendation to use platinum-containing drugs for neoadjuvant therapy in patients with resectable stage IIIA NSCLC has been based on the meta-analysis of a few clinical trials conducted over the past 20 years $(11,12)$.

In contrast to traditional platinum-based chemotherapy, novel drugs that regulate the immune system of patients to release their own anti-tumor immunity have been gradually applied to modern treatment strategies for NSCLC. Among them, the regulatory immune-inhibitory pathway of programmed death receptor 1 (PD-1) and its ligand (PD-L1) has attracted special attention. The expression of these molecules can be observed on the surface of different types of body cells, including PD-L1 which is mainly expressed on the surface of tumor cells and PD-1 which is expressed on the surface of activated T-cells. When PD-1 and PD-L1 combine, they activate the regulatory immuneinhibitory pathway, thereby inhibiting the function of selfactivated T-cells which results in a decline in the body's anti-tumor immune response and loss of its killing effect on tumor cells $(13,14)$.

Clinical trials including the CheckMate and KEYNOTE-trials have shown that inhibitors which block PD-1 and/or PD-L1 immune checkpoints have benefited patients with stage IV NSCLC (15-17). These encouraging results for the treatment of patients with advanced NSCLC provide a strong and powerful theoretical basis for the exploration of immune checkpoint inhibitors (ICIs) in the neoadjuvant treatment of NSCLC. Currently, multiple clinical trials related to neoadjuvant immunotherapy have achieved certain phase results, such as CheckMate-159, LCMC3, NADIM, and NeoStar (18-21). The CheckMate-159 trial is the first prospective research to explore the feasibility and safety of ICIs neoadjuvant therapy in untreated and resectable stage I-IIIA NSCLC patients. A total of 22 eligible patients were recruited, of which $20(90 \%)$ underwent surgery after two treatment cycles of the neoadjuvant drug nivolumab, and nine patients (45\%) achieved the major pathological response (MPR) (18). While the sample size of that prospective trial was small, it confirmed the safety of neoadjuvant monotherapy for NSCLC, laying a solid foundation for subsequent research $(20,22)$. The purpose of the clinical phase II NADIM trial was to explore the feasibility and safety of nivolumab combined with paclitaxel and carboplatin in neoadjuvant and adjuvant treatment of patients with resectable stage IIIA NSCLC. Among the 46 patients who received combined neoadjuvant therapy, 41 (89\%) patients underwent $\mathrm{R} 0$ resection, and postoperative pathology found that $34(83 \%)$ of 41 achieved MPR, and the pathological complete response (pCR) reached 63\% (20). In 2020, Jia and his colleagues published a meta-analysis based on existing clinical trial data, which also verified the safety and feasibility of neoadjuvant immunotherapy for treatment of resectable NSCLC (23).

However, the ongoing and completed clinical trials of neoadjuvant immune monotherapy for NSCLC to date 
have concerned patients with stage I to stage IIIA disease, and there is a lack of research that only selects clinical stage IIIA patients for neoadjuvant immune combined chemotherapy like the NADIM clinical trial. On this basis, we conducted a retrospective study of neoadjuvant therapy for stage IIIA NSCLC and compared the perioperative safety and feasibility outcomes that follow neoadjuvant immune monotherapy or neoadjuvant chemotherapy. We present the following article in accordance with the STROBE reporting checklist (available at http://dx.doi. org/10.21037/atm-21-1141).

\section{Methods}

\section{Patient selection}

This study was carried out in accordance with the Helsinki Declaration (as revised in 2013) and ethics approval was obtained from the Institutional Review Board of the authors [No. QYFYKY (2018-10-11-2)]. All participants gave written informed consent for their participation.

We retrospectively collected and analyzed patients with stage IIIA (N2) NSCLC who received neoadjuvant therapy at the Affiliated Hospital of Qingdao University from January 2019 to December 2020. Based on the 8th edition TNM staging of lung cancer of the American Joint Committee on Cancer (AJCC), the clinical stage of patients before neoadjuvant treatment was cT1-2N2M0 (24). The inclusion criteria were as follows: (I) all patients were 18 years of age or older and had an Eastern Cooperative Oncology Group (ECOG) performance status (PS) of 0-1 (25); (II) all patients were diagnosed with NSCLC by pathological or histological examination before neoadjuvant treatment; (III) all patients had normal organ function including adequate cardiopulmonary function, and could undergo surgical resection after discussion with a multidisciplinary team (MDT); (IV) the clinical stage of all patients was confirmed before neoadjuvant therapy based on the results of positron emission tomography with computed tomography (PET-CT) of the whole body, contrastenhanced CT, and magnetic resonance imaging (MRI) of the brain; (V) N2 lymph node involvement was sought by biopsy using mediastinoscopy and/or endobronchial ultrasonography (EBUS). When biopsy could not confirm this, the necessary condition was that the diameter of the $\mathrm{N} 2$ lymph node was greater than $1 \mathrm{~cm}(>1 \mathrm{~cm}$ in contrastenhanced CT image).

The exclusion criteria were any of the following: (I) patients had received any other anti-tumor related treatment before admission; (II) patients with distant metastasis; (III) patients with other significant malignant tumors; (IV) patients with known EGFR wild-type mutations or ALK translocation rearrangements.

\section{Neoadjuvant procedures}

After MDT consultation and evaluation, eligible patients were randomly allocated to a neoadjuvant immunotherapy group and neoadjuvant chemotherapy group. Patients in the immunotherapy group received the drug nivolumab $(3 \mathrm{mg} / \mathrm{kg}$ ) intravenously on day 1 of each 21 -day cycle $(\mathrm{q} 3 \mathrm{w})$, for two cycles (day 1, day 22), and in the chemotherapy group gemcitabine $\left(1,000 \mathrm{mg} / \mathrm{m}^{2}\right)$ was given on days 1 and 8 followed by cisplatin $\left(80 \mathrm{mg} / \mathrm{m}^{2}\right)$ on day 1 of each 21 -day cycle (q3w), for two cycles.

All patients underwent laboratory blood tests before every 3 -week treatment cycle to monitor complete blood counts and biochemical parameters. All patients were monitored for adverse advents throughout the course of treatment based on the National Cancer Institute Common Terminology Criteria for Adverse Events, Version 4.0 (NCI-CTCAEV4.0) (26). Contrast-enhanced CT of the chest was repeated to assess tumor response following two cycles of neoadjuvant therapy and within 7 days before surgery. All tumor size changes were evaluated according to the Response Evaluation Criteria in solid Tumors (RECIST), version 1.1 (27). If the tumor progressed during the evaluation, the patient did not undergo surgery and was switched to other treatment.

\section{Surgical procedures}

Following the completion of neoadjuvant treatment, every patient who met the eligibility criteria underwent radical surgery between 21-28 days after the end of the second treatment cycle.

All operations were performed under general anesthesia by the same surgical team with a video-assisted thoracoscopic surgery (VATS) system and using one-lung ventilation and double-lumen endotracheal intubation. During the operation, the patient adopted the contralateral position and with the chest raised, a small $1.5 \mathrm{~cm}$ opening was cut at the 7 th or 8 th intercostal space of the mid-axillary line as the lens port, and a poking card inserted. In addition, an operation port was located in the fourth intercostal space 
of the front axillary line with an incision width of $3-5 \mathrm{~cm}$. Surgical procedures for stage IIIA (N2) disease included the removal of primary lung cancer and a systemic LN dissection of the ipsilateral hilum and mediastinum. The scope of surgical resection was determined by the surgeon according to the individual circumstances of the patient. Regardless of the surgical method, all patients routinely underwent mediastinal LN dissection, which contains groups 4 to 12 lymph nodes $(5,6,7,8,9,10 \mathrm{~L}$, and 11L) on the left-side and 2 to 4 and 7 to 12 lymph nodes (2R, $4 \mathrm{R}$, $7,8,9,10 \mathrm{R}$, and $11 \mathrm{R}$ ) on the right-side. All LNs surgically removed were pathologically examined and each classified by anatomical location using the numbering system described in the Mountain-Dresler modification of the American Thoracic Society (MD-ATS) (28).

The surgical indicators of the two groups, including operative time, estimated blood loss, complete resection (R0) rate, and procedure and condition of lymph node dissection were first recorded. The postoperative related indicators of the patient, including pathologic remission and TNM down-staging were then recorded. Finally, morbidity, mortality, and surgical complications were continuously monitored during the first 30 days after surgery.

\section{Pathological assessments}

The objective pathological response was evaluated by pathologists at the author's institution based on measuring the percentage of residual viable tumor cells in resected primary tumor, then confirmed by two additional blinded pathologists. We defined the MPR as the presence of $10 \%$ or less viable tumor cells in resected primary tumor, while the pCR was defined as the tumor without any viable tumor cells in the removed lung cancer specimens and all sampled regional lymph nodes (29-31).

\section{Objectives and endpoints of the study}

The primary objectives were to assess the safety and feasibility of a neoadjuvant application of nivolumab or chemotherapy (GP: gemcitabine and cisplatin) and to evaluate the efficacy of neoadjuvant therapy on the clinical and pathologic tumor response.

The primary endpoint was the risk of major complications within 30 days after surgery and secondary endpoints were interval to surgery and 30-day mortality (32). According to the Clavien-Dindo classification system, major complications were defined as grade $\geq 3$.

\section{Statistical analysis}

The mean and $95 \%$ confidence interval (CI) were used to describe continuous variables, while categorical variables were expressed in counts and percentages. The normality of individual parameter distributions was assessed with the Shapiro-Wilk test and comparisons between two groups of continuous variables were made using Student's $t$ test or Wilcoxon rank-sum test. The Chi-square test or Fisher's exact test was used to compare categorical variables. All statistical analyses were performed using R 4.0.3 (The R Development Core Team, Vienna, Austria) and all statistical tests were two-sided tests. A $\mathrm{P}<0.05$ was considered to indicate a statistically significance difference.

\section{Results}

From January 2019 to December 2020, a total of 107 eligible patients with stage IIIA (N2) NSCLC received neoadjuvant treatments, of whom 25 received neoadjuvant immunotherapy and 82 received neoadjuvant chemotherapy (Figure 1). The baseline clinicopathologic features of patients in the two treatment groups were well balanced (Table 1). Only $1(4 \%)$ patient in the immunotherapy group and $5(6 \%)$ in the chemotherapy group had mediastinal lymph node involvement that could not be reached for biopsy.

\section{Tumor responses after neoadjuvant treatment}

The radiologic response after at least one cycle of preoperative neoadjuvant therapy of nivolumab or GP are shown in Table 2. Of the 25 patients in the neoadjuvant immunotherapy group who had clinical tumor results that could be evaluated, 8 patients (32\%) had a partial response (PR), 16 (64\%) had stable disease (SD), and 1 (4\%) had progressive disease $(\mathrm{PD})$. However, a complete response (CR) was seen in $8(9.8 \%)$ patients after neoadjuvant chemotherapy, and a PR was seen in 36 (43.9\%). When induction strategies were compared, we found no significant statistical differences between the two groups $(\mathrm{P}=0.149)$.

\section{Treatment-related adverse events}

All 107 patients receiving neoadjuvant treatments were evaluated for safety and the results are summarized in Table 3.

\section{Immune-related adverse events}

In the neoadjuvant immunotherapy group, all 25 patients 


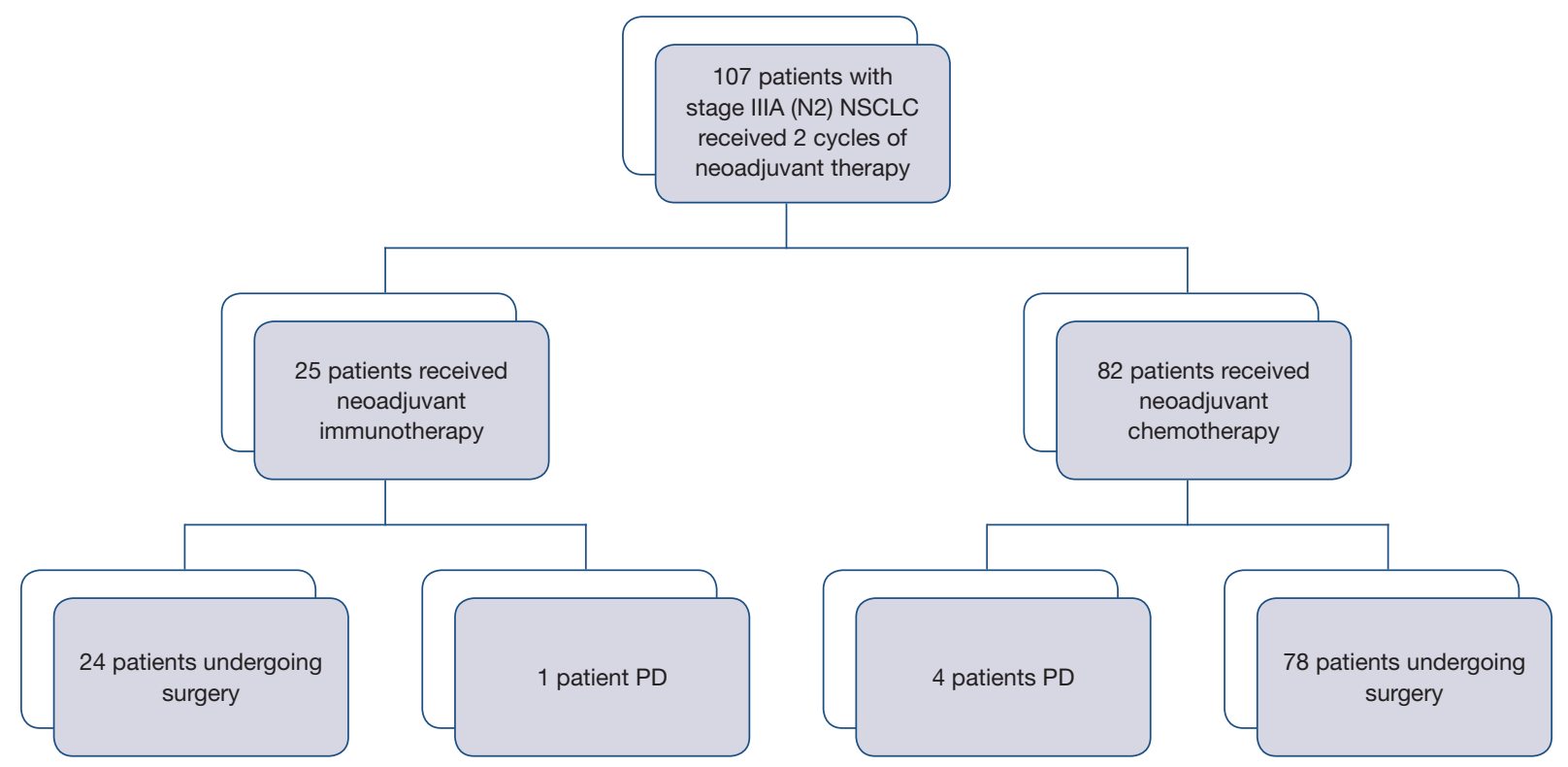

Figure 1 A schematic of the study subject selection process. NSCLC, non-small cell lung cancer; PD, progressive disease.

received two cycles of treatment before surgery. Overall, the incidence of immune toxicity in the preoperative setting was low with $2(8 \%)$ patients in each experiencing grade 2 and grade 3 hepatitis, two patients with grade 2 dermatitis, and one patient each with grade 2 pneumonia, grade 1 nephritis, and grade 3 colitis. Therefore, immune-related adverse events of any grade occurred in seven of 25 patients (28\%; 95\% CI, 12.1 to 49.4 ), and only 3 (12\%) events were of grade 3 or higher. None of these adverse events delayed the next operation for more than 2 weeks.

\section{Chemotherapy-related adverse events}

In the chemotherapy group, $80(97.6 \%)$ of 82 patients received two cycles of neoadjuvant chemotherapy, and the reason for not completing treatment was that two (2.4\%) patients had disease progression after one cycle of treatment. Treatment-related adverse events were seen in 77 (93.9\% CI, 86.3 to 98$)$ of 82 and 17 (20.7\% CI, 12.6 to 31.1 ) of 82 patients had adverse events of grade 3 or higher. The most common grade 1 or 2 non-hematologic treatment-related adverse events were asthenia or fatigue [35 (42.7\%) of 82 patients], alopecia [32 (39\%)], myalgia [22 $(26.8 \%)]$, arthralgia [21 (25.6\%)], diarrhoea [20 (24.4\%)], skin disorders [18 (22\%)], neurotoxicity [16 (19.5\%)], constipation [15 (18.3\%)], and vomiting [14 (17.1\%)]. Leukopenia [24 (29.3\%)], thrombocytopenia [18 (22\%)], and anemia [12 (14.6\%)] were the most common grade 1 or
2 hematologic treatment-related adverse events. The most common treatment-related grade 3 or higher adverse events were leukopenia [5 (6.1\%) of 82 patients] and neurotoxicity [4 (4.9\%)]. Nonetheless, none of the adverse events of any grade reported during neoadjuvant chemotherapy caused the patient to delay surgery or die.

\section{Surgical procedures and outcomes}

Table 4 summarizes surgical procedures and outcomes of surgery. Of the 107 patients assessable for resection, 102 underwent surgical resection, including 24 in the neoadjuvant immunotherapy group and 78 in the neoadjuvant chemotherapy group. The mean interval between completion of the second dose of drug therapy and surgery was 29.2 days (95\% CI, 27.1 to 31.4 days) in the immunotherapy group and 28.7 days ( $95 \%$ CI, 27.6 to 29.8 days) in those receiving chemotherapy. By comparison, the mean interval to surgery had no clinical significance $(\mathrm{P}=0.656)$.

The surgical procedures performed were lobectomy (79.2\% versus 75.6 ), bilobectomy ( $12.5 \%$ versus $11.5 \%)$, pneumonectomy ( $4.2 \%$ versus $6.4 \%$ ), and other procedures ( $4.2 \%$ versus $6.4 \%)$. In the two groups, complete resections (R0) in those receiving surgery occurred in $95.8 \%$ versus $84.6 \%$. There was no significant statistical difference in operation time $(\mathrm{P}=0.854)$ and estimated blood loss $(\mathrm{P}=0.684)$, in the two groups. Similarly, there was no 
Table 1 Baseline clinicopathologic characteristics of patients

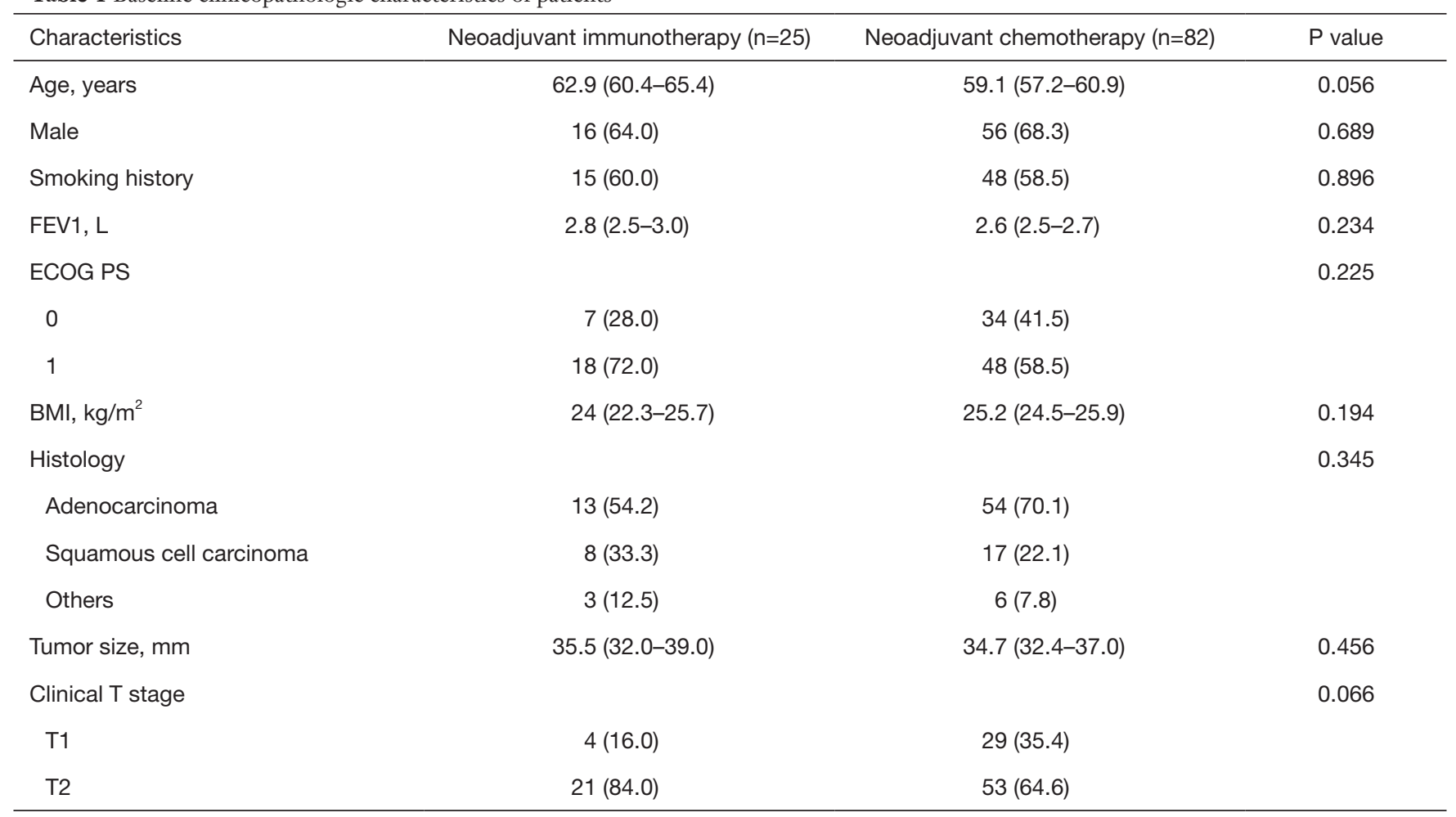

Data are presented as no. (\%) or mean (95\% confidence interval). FEV1, forced expiratory volume during the first second; BMI, body mass index; ECOG PS, Eastern Cooperative Oncology Group Performance Status.

Table 2 Tumor responses following neoadjuvant treatment

\begin{tabular}{lcc}
\hline Characteristics & Neoadjuvant immunotherapy $(\mathrm{n}=25)$ & Neoadjuvant chemotherapy $(\mathrm{n}=82)$ \\
\hline Radiologic responses & 0 & $8(9.8)$ \\
Complete response & $8(32.0)$ & $36(43.9)$ \\
Partial response & $16(64.0)$ & $34(41.4)$ \\
Stable disease & $1(4.0)$ & $4(4.9)$ \\
Progressive disease & & 0.149 \\
\hline
\end{tabular}

Data are presented as no. (\%) unless otherwise noted. Based on Response Evaluation Criteria in solid Tumors (RECIST) version 1.1 and classification.

statistical difference in the probability of postoperative complications of grade 3 or higher between the two groups $(\mathrm{P}=0.757)$. The most common grade 3 or 4 postoperative complications (within 30 days) were atrial fibrillation, followed by subcutancous emphysema and prolonged air leak, and other events occurring in one patient each.

\section{Pathologic response}

Table 5 summarizes pathologic response outcomes.
According to postoperative pathological results, in the neoadjuvant immunotherapy group, $9(37.5 \%)$ of 24 patients who underwent surgery had a MPR, and $1(4.2 \%)$ patient had a complete pathological response. In the neoadjuvant chemotherapy group, of the 78 patients who underwent resection, 10 (12.8\%) achieved a MPR, and 2 (2.6\%) had a complete pathological response. There was a significant difference in the pathological remission of the two groups $(\mathrm{P}=0.023)$.

Among the 24 patients who underwent resection 
Table 3 Treatment-related adverse events during neoadjuvant treatment

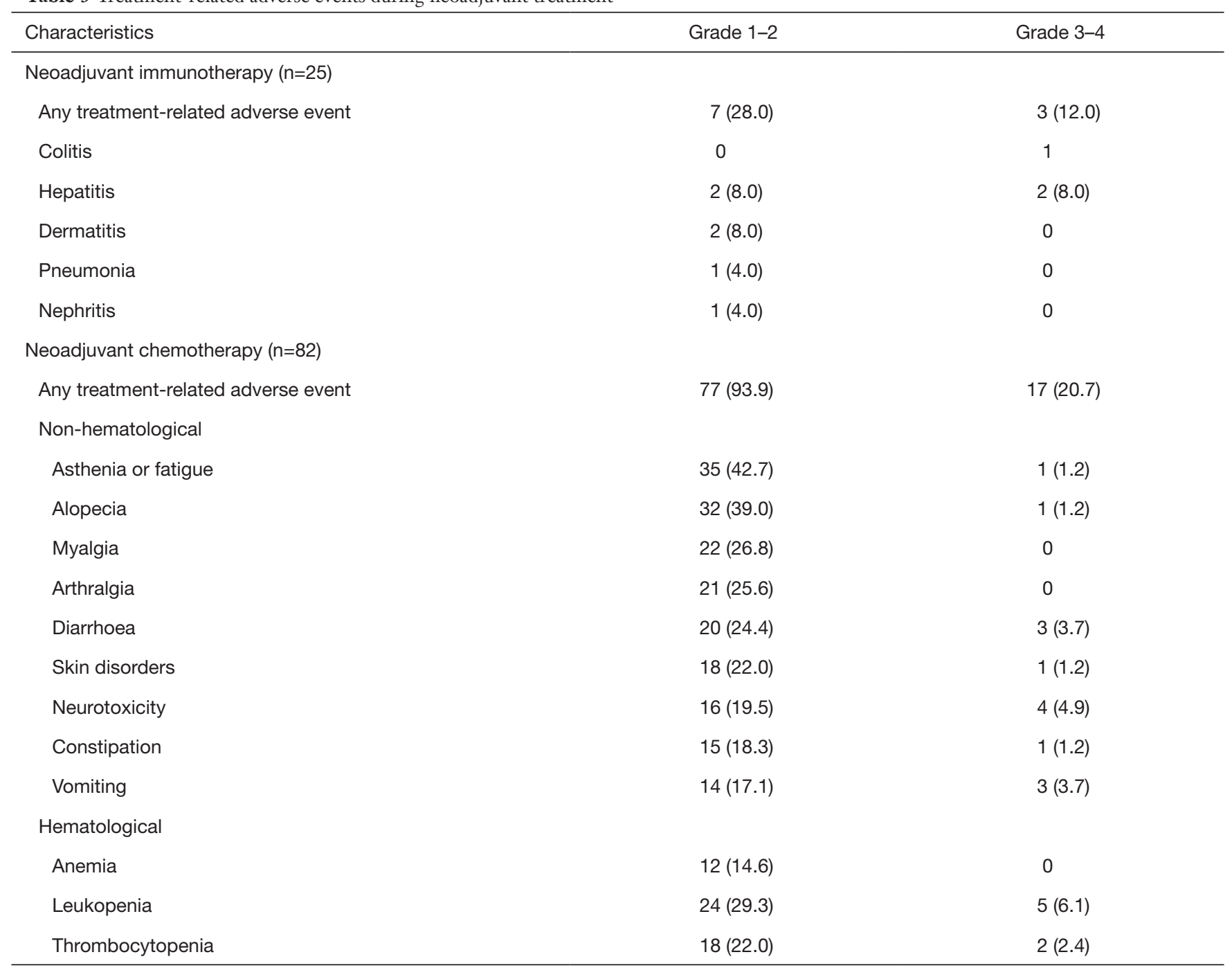

Data are presented as no. (\%) unless otherwise noted. Patients might have experienced more than one adverse event.

following nivolumab therapy, pathological down-staging from the pretreatment clinical stage occurred in 11 (45.8\%) patients. In neoadjuvant chemotherapy group, $40(51.3 \%)$ patients reached the pathological stage of the clinical disease stage. However, no significant associations were identified between any clinical parameter and pathological downstaging $(\mathrm{P}=0.641)$.

\section{Discussion}

This study was a single-center retrospective study to compare the perioperative safety and feasibility outcomes of a neoadjuvant immunotherapy group and a neoadjuvant chemotherapy group in the treatment of resectable stage

\section{IIIA (N2) NSCLC.}

The results show that of the 107 patients receiving neoadjuvant therapy, 102 achieved the primary and secondary endpoints. One patient in the neoadjuvant immunotherapy group and four in the neoadjuvant chemotherapy group did not undergo surgery because their disease progressed, and they were switched to treatment with other strategies. There was no significant statistical difference between the two groups in other clinical indicators except for pathological remission. Further comparison of the postoperative pathological remission of the two groups showed that the immunotherapy group had a higher MPR. Some research suggests that the MPR may be related to the long-term survival of patients and that it will become an indicator of 
Table 4 Surgical procedures and outcomes

\begin{tabular}{|c|c|c|c|}
\hline Characteristics & Neoadjuvant immunotherapy $(\mathrm{n}=24)$ & Neoadjuvant chemotherapy $(\mathrm{n}=78)$ & $P$ value \\
\hline Procedure & & & 1.000 \\
\hline Lobectomy & $19(79.2)$ & $59(75.6)$ & \\
\hline Bilobectomy & $3(12.5)$ & $9(11.5)$ & \\
\hline Other & $1(4.2)$ & $5(6.4)$ & \\
\hline Outcome & & & 0.385 \\
\hline Complete resection (R0) & $23(95.8)$ & $66(84.6)$ & \\
\hline Incomplete resection (R1) & $1(4.2)$ & $10(12.8)$ & \\
\hline Estimated blood loss, $\mathrm{mL}$ & $91.7(67.4-115.9)$ & $86.7(75.2-98.1)$ & 0.684 \\
\hline Number of lymph nodes dissected & $17.3(14.7-19.9)$ & $19.9(18.3-21.5)$ & 0.126 \\
\hline Length of hospital stay, days & $5.8(5.3-6.3)$ & $5.8(5.4-6.2)$ & 0.980 \\
\hline Thirty-day deaths & 0 & $1(1.3)$ & 1.000 \\
\hline Complications grade $\geq 3$ & 3 & 13 & 0.757 \\
\hline Atrial fibrillation & $1(4.2)$ & $5(6.4)$ & \\
\hline Prolonged air leak & $1(4.2)$ & $2(2.6)$ & \\
\hline Pneumothorax & 0 & $1(1.3)$ & \\
\hline
\end{tabular}

Data are presented as no. (\%) or mean (95\% confidence interval).

the survival time of patients in the future, but more research is required to confirm this $(33,34)$.

The concept of neoadjuvant chemotherapy, which refers to systemic or local chemotherapy given before local treatment of malignant tumors (surgery or radiotherapy) and is also used to distinguish postoperative adjuvant chemotherapy, was first proposed by Frei and his colleagues in 1982. Until now, researchers have paid close attention to neoadjuvant immunotherapy with anti-PD-1 and antiPD-L1 ICIs. However, this comprehensive treatment plan that is still in the clinical trials stage and full evaluation of its safety and effectiveness is critical.

Since the role of neoadjuvant therapy in the treatment of NSCLC is a relatively complex problem involving many factors, this study only sought to analyze safety during a limited period, the perioperative period.

Most of the time we focus more on NSCLC, but it should not be overlooked that about $13-15 \%$ of patients are small cell lung cancer (SCLC) (35). SCLC is one of the most common malignant tumors that seriously threaten the life and health of patients, and can be divided into limited-stage SCLC (LS-SCLC) and extensive-stage SCLC (ES-SCLC). Chemotherapy combined radiotherapy is still the standard treatment for the vast majority of LSSCLC patients. At the 2018 World Conference on Lung Cancer, the IMpower-133 study published has achieved an unprecedented breakthrough, opening a new chapter in the treatment of ES-SCLC (36). However, the treatment strategy of SCLC is still worth exploring.

There are several limitations to this study. Firstly, its 
Table 5 Pathologic response

\begin{tabular}{|c|c|c|c|}
\hline Characteristics & Neoadjuvant immunotherapy $(n=24)$ & Neoadjuvant chemotherapy $(n=78)$ & $P$ value \\
\hline Major pathological response & $9(37.5)$ & $10(12.8)$ & \\
\hline Complete pathological response & $1(4.2)$ & $2(2.6)$ & \\
\hline Pathological T down-staging & $9(37.5)$ & $28(35.9)$ & 0.886 \\
\hline
\end{tabular}

Data are presented as no. (\%) unless otherwise noted.

retrospective nature produces two sets of data with selective deviations, which increase the deviation of research results. Therefore, we need to conduct forward-looking randomized control studies in the future. Secondly, the study was a small sample study conducted in a single medical center and will require a large sample size multi-center study in the future to confirm the results. At the same time, this study did not include patients with genetic variations in the study population, whether it will affect the neoadjuvant therapy response and overall survival of patients needs to be further explored. In addition, this study mainly demonstrates the perioperative safety and feasibility outcomes of neoadjuvant treatment for stage IIIA (N2) NSCLC after surgery. We will continue to follow-up and hope to provide findings pertinent to long-term efficacy such as tumor recurrence, metastasis, or 5 -year survival rate, in the future.

\section{Conclusions}

When comparing the perioperative safety and feasibility results of neoadjuvant immunotherapy and neoadjuvant chemotherapy for the treatment of stage IIIA (N2) NSCLC, we found that most patients from each group achieved the primary and secondary endpoints of the study. However, by analyzing the research data, it can be seen that the two groups of patients have significant statistical differences in the main pathological response.

\section{Acknowledgments}

Funding: This research was funded by Key R \& D programs in Shandong Province (grant number: 2018GSF118119).

\section{Footnote}

Reporting Checklist: The authors have completed the
STROBE reporting checklist. Available at http://dx.doi. org/10.21037/atm-21-1141

Data Sharing Statement: Available at http://dx.doi. org/10.21037/atm-21-1141

Conflicts of Interest: All authors have completed the ICMJE uniform disclosure form (available at http://dx.doi. org/10.21037/atm-21-1141). The authors have no conflicts of interest to declare.

Ethical Statement: The authors are accountable for all aspects of the work in ensuring that questions related to the accuracy or integrity of any part of the work are appropriately investigated and resolved. This study was carried out in accordance with the Helsinki Declaration (as revised in 2013) and ethics approval was obtained from the Institutional Review Board of the authors [No. QYFYKY (2018-10-11-2)]. All participants gave written informed consent for their participation.

Open Access Statement: This is an Open Access article distributed in accordance with the Creative Commons Attribution-NonCommercial-NoDerivs 4.0 International License (CC BY-NC-ND 4.0), which permits the noncommercial replication and distribution of the article with the strict proviso that no changes or edits are made and the original work is properly cited (including links to both the formal publication through the relevant DOI and the license). See: https://creativecommons.org/licenses/by-nc-nd/4.0/.

\section{References}

1. Bade BC, Dela Cruz CS. Lung Cancer 2020: Epidemiology, Etiology, and Prevention. Clin Chest Med 2020;41:1-24. 
2. Siegel RL, Miller KD, Jemal A. Cancer statistics, 2020. CA Cancer J Clin 2020;70:7-30.

3. Sosa MS, Bragado P, Aguirre-Ghiso JA. Mechanisms of disseminated cancer cell dormancy: an awakening field. Nat Rev Cancer 2014;14:611-22.

4. Travis WD, Brambilla E, Nicholson AG, et al. The 2015 World Health Organization Classification of Lung Tumors: Impact of Genetic, Clinical and Radiologic Advances Since the 2004 Classification. J Thorac Oncol 2015;10:1243-60.

5. Goldstraw P, Chansky K, Crowley J, et al. The IASLC Lung Cancer Staging Project: Proposals for Revision of the TNM Stage Groupings in the Forthcoming (Eighth) Edition of the TNM Classification for Lung Cancer. J Thorac Oncol 2016;11:39-51.

6. De Marinis F, Gebbia V, De Petris L. Neoadjuvant chemotherapy for stage IIIA-N2 non-small cell lung cancer. Ann Oncol 2005;16 Suppl 4:iv116-122.

7. Goldstraw P, Ball D, Jett JR, et al. Non-small-cell lung cancer. Lancet 2011;378:1727-40.

8. Preoperative chemotherapy for non-small-cell lung cancer: a systematic review and meta-analysis of individual participant data. Lancet 2014;383:1561-71.

9. Felip E, Rosell R, Maestre JA, et al. Preoperative Chemotherapy Plus Surgery Versus Surgery Plus Adjuvant Chemotherapy Versus Surgery Alone in Early-Stage NonSmall-Cell Lung Cancer. J Clin Oncol 2010;28:3138-45.

10. Wu YL, Yang XN, Zhong W, et al. Multi-centre randomized controlled study comparing adjuvant vs neoadjuvant chemotherapy with docetaxel plus carboplatin in resectable stage IB to IIIA NSCLC: final results of CSLC0501. Ann Oncol 2016;27:VI407.

11. Casiraghi M, Guarize J, Sandri A, et al. Pneumonectomy in Stage IIIA-N2 NSCLC: Should It Be Considered After Neoadjuvant Chemotherapy? Clin Lung Cancer 2019;20:97-106.e1.

12. Douillard J-Y, Tribodet H, Aubert D, et al. Adjuvant cisplatin and vinorelbine for completely resected non-small cell lung cancer: subgroup analysis of the Lung Adjuvant Cisplatin Evaluation. J Thorac Oncol 2010;5:220-8.

13. Santarpia M, Aguilar A, Chaib I, et al. Non-Small-Cell Lung Cancer Signaling Pathways, Metabolism, and PD-1/ PD-L1 Antibodies. Cancers (Basel) 2020;12:1475.

14. Herbst RS, Baas P, Kim D-W, et al. Pembrolizumab versus docetaxel for previously treated, PD-L1-positive, advanced non-small-cell lung cancer (KEYNOTE-010): a randomised controlled trial. Lancet 2016;387:1540-50.

15. Antonia SJ, Borghaei H, Ramalingam SS, et al. Four-year survival with nivolumab in patients with previously treated advanced non-small-cell lung cancer: a pooled analysis.

Lancet Oncol 2019;20:1395-408.

16. Herbst RS, Garon EB, Kim DW, et al. Long-Term Outcomes and Retreatment Among Patients With Previously Treated, Programmed Death-Ligand 1-Positive, Advanced Non-Small-Cell Lung Cancer in the KEYNOTE-010 Study. J Clin Oncol 2020;38:1580-90.

17. Paz-Ares L, Vicente D, Tafreshi A, et al. A Randomized, Placebo-Controlled Trial of Pembrolizumab Plus Chemotherapy in Patients With Metastatic Squamous NSCLC: Protocol-Specified Final Analysis of KEYNOTE-407. J Thorac Oncol 2020;15:1657-69.

18. Forde PM, Chaft JE, Smith KN, et al. Neoadjuvant PD-1 Blockade in Resectable Lung Cancer. N Engl J Med 2018;378:1976-86.

19. Rusch VW, Chaft JE, Johnson B, et al. Neoadjuvant atezolizumab in resectable non-small cell lung cancer (NSCLC): Initial results from a multicenter study (LCMC3). J Clin Oncol 2018;36:8541-.

20. Provencio M, Nadal E, Insa A, et al. Neoadjuvant chemotherapy and nivolumab in resectable non-small-cell lung cancer (NADIM): an open-label, multicentre, singlearm, phase 2 trial. Lancet Oncol 2020;21:1413-22.

21. Cascone T, William WN, Weissferdt A, et al. Neoadjuvant nivolumab $(\mathrm{N})$ or nivolumab plus ipilimumab (NI) for resectable non-small cell lung cancer (NSCLC): Clinical and correlative results from the NEOSTAR study. J Clin Oncol 2019;37:abstr 8504.

22. Gandhi L, Rodríguez-Abreu D, Gadgeel S, et al. Pembrolizumab plus Chemotherapy in Metastatic NonSmall-Cell Lung Cancer. N Engl J Med 2018;378:2078-92.

23. Jia XH, Xu H, Geng LY, et al. Efficacy and safety of neoadjuvant immunotherapy in resectable nonsmall cell lung cancer: A meta-analysis. Lung Cancer 2020;147:143-53.

24. Detterbeck FC, Boffa DJ, Kim AW, et al. The Eighth Edition Lung Cancer Stage Classification. Chest 2017;151:193-203.

25. Oken MM, Creech RH, Tormey DC, et al. Toxicity and response criteria of the Eastern Cooperative Oncology Group. Am J Clin Oncol 1982;5:649-55.

26. National Institutes of Health National Cancer Institute. Common Terminology Criteria for Adverse Events (CTCAE) v4.0. 2009.

27. Eisenhauer EA, Therasse P, Bogaerts J, et al. New response evaluation criteria in solid tumours: revised RECIST guideline (version 1.1). Eur J Cancer 2009;45:228-47. 
28. Mountain CF, Dresler CM. Regional lymph node classification for lung cancer staging. Chest 1997;111:1718-23.

29. Hellmann MD, Chaft JE, William WN, et al. Pathological response after neoadjuvant chemotherapy in resectable non-small-cell lung cancers: proposal for the use of major pathological response as a surrogate endpoint. Lancet Oncol 2014;15:e42-e50.

30. Qu Y, Emoto K, Eguchi T, et al. Pathologic Assessment After Neoadjuvant Chemotherapy for NSCLC: Importance and Implications of Distinguishing Adenocarcinoma From Squamous Cell Carcinoma. J Thorac Oncol 2019;14:482-93.

31. A WDT, B SD, C IW, et al. IASLC Multidisciplinary Recommendations for Pathologic Assessment of Lung Cancer Resection Specimens After Neoadjuvant Therapy. J Thorac Oncol 2020;15:709-40.

32. Dindo D, Demartines N, Clavien PA. Classification of surgical complications: a new proposal with evaluation in

Cite this article as: Huang Z, Wu Z, Qin Y, Zhao Y, Xuan Y, Qiu T, Liu A, Dong Y, Su W, Du W, Yun T, Wang L, Liu D, Sun L, Jiao W. Perioperative safety and feasibility outcomes of stage IIIA-N2 non-small cell lung cancer following neoadjuvant immunotherapy or neoadjuvant chemotherapy: a retrospective study. Ann Transl Med 2021;9(8):685. doi: 10.21037/atm-211141 a cohort of 6336 patients and results of a survey. Ann Surg 2004;240:205-13.

33. Weissferdt A, Pataer A, Vaporciyan AA, et al. Agreement on Major Pathological Response in NSCLC Patients Receiving Neoadjuvant Chemotherapy. Clin Lung Cancer 2020;21:341-8.

34. Schreiner W, Dudek W, Rieker RJ, et al. Major Pathologic Response after Induction Therapy Has a Long-Term Impact on Survival and Tumor Recurrence in Stage IIIA/ B Locally Advanced NSCLC. Thorac Cardiovasc Surg 2020;68:639-45.

35. Sen T, Gay CM, Byers LA. Targeting DNA damage repair in small cell lung cancer and the biomarker landscape. Transl Lung Cancer Res 2018;7:50-68.

36. Horn L, Mansfield AS, Szczęsna A, et al. First-Line Atezolizumab plus Chemotherapy in Extensive-Stage Small-Cell Lung Cancer. N Engl J Med 2018;379:2220-9.

(English Language Editor: B. Draper) 